\title{
NON-POSITIVE SEMIGROUP DYNAMICS IN CONTINUOUS VARIABLE MODELS
}

\author{
F. BENATTI \\ Dipartimento di Fisica Teorica, Università di Trieste, \\ Strada Costiera 11, 34014 Trieste, Italy \\ and Istituto Nazionale di Fisica Nucleare, Sezione di Trieste \\ benatti@ts.infn.it \\ R. FLOREANINI \\ Istituto Nazionale di Fisica Nucleare, Sezione di Trieste, \\ Dipartimento di Fisica Teorica, Università di Trieste, \\ Strada Costiera 11, 34014 Trieste, Italy \\ florean@ts.infn.it
}

\begin{abstract}
Non-positive, Markovian semigroups are sometimes used to describe the time evolution of subsystems immersed in an external environment. A widely adopted prescription to avoid the appearance of negative probabilities is to eliminate from the admissible initial conditions those density matrices that would not remain positive by the action of the semigroup dynamics. Using a continuous variable model, we show that this procedure leads to physical inconsistencies when two subsystems are considered and their initial state is entangled.
\end{abstract}

\section{Introduction}

The time evolution of a subsytem in weak interaction with an external environment can be modelled in terms of Markovian (i.e. memoryless) dynamical semigroups; these are families $\left\{\gamma_{t}\right\}$ of one-parameter (=time) maps acting on the states of the subsystem, typically described by density matrices, and obeying the forward in time composition law, $\gamma_{t} \circ \gamma_{s}=\gamma_{t+s}, t, s \geq 0$. Such a description is very general and have been successfully used to describe various effects in open system dynamics, in quantum optics, quantum chemistry and atomic physics 1 -12

Nevertheless, the derivation of such time evolutions from the microscopic interaction of the subsystem with the environment is often based on ad hoc approximations, justified a posteriori with various physical considerations. As a result, the obtained time evolutions might not be fully consistent: in particular, such dynamics do not in general preserve the positivity of the reduced density matrix. Exceptions to this general result are obtained through the use of rigorous mathematical treatments $1-5$

These inconsistencies are either dismissed as irrelevant for all practical purposes 13 or cured by adopting further ad hoc prescriptions $14-16$ In the lat- 
ter case, the general attitude is to restrict the action of the non-positive dynamical maps to a subset of all possible initial reduced density matrices, those for which the time-evolution remains positive. This is equivalent to a suitable selection of the initial conditions for the starting state of the subsystem, a procedure sometimes referred to as "slippage of the initial conditions". On physical grounds, this effect is viewed as the consequence of the short-time correlations in the environment, that have not been properly taken into account in the derivation of the Markovian limit of the evolution, usually based on weak-coupling limit techniques that get rid of transient effects.

In the following, we shall reexamine this widely used prescription to cure possible inconsistencies produced by non-positive, Markovian evolutions, and point out further potential problems of this approach. Some results on finite-dimensional systems have been reported in Ref.[17, 18]. In this note we shall instead examine the case of simple infinite-dimensional systems. More specifically, we shall study the behaviour of two independent bosonic oscillators, one evolving with a dissipative Markovian semigroup, while the other with a standard unitary dynamics. We shall limit our considerations to the phenomenologically relevant set of Gaussian states $19-27$ and to dissipative dynamics that preserve this set, the so-called quasi-free semigroups. $27-29$

Although very simple, this settings actually corresponds to a specific physical situation in quantum optics $30-33$, that of two coherent states travelling in optical fibers, of which only one is subjected to noise, producing dissipative phenomena, while the other gives rise only to unitary, standard birefringence effects. The considerations that follow may therefore have direct phenomenological and experimental relevance.

We shall explicitly show that when the initial state of the two oscillators is entangled, redefining the initial conditions to make positive the single system timeevolution is not enough to cure all possible inconsistencies of the two-system dynamics. Therefore, in order to have a physically acceptable time evolution for the two subsystems in presence of entanglement, the above mentioned procedure of restricting initial conditions should be tested against the presence of non-classical correlations.

\section{Single Mode Dissipative Dynamics}

We shall first study the dynamics of a single oscillator in weak interaction with an external environment, so that its reduced time evolution can be well represented by a Markovian semigroup. 26 The states of this system will be represented by a density matrix $\rho$, i.e. by a positive Hermitian operator, with unit trace, acting on a bosonic Hilbert space $\mathcal{H}$. It can be identified with a Fock space, generated from the vacuum state through the action of polynomials in the creation $a^{\dagger}$ and annihilation $a$ operators; these operators obey the standard bosonic oscillator algebra: $\left[a, a^{\dagger}\right]=1$, $\left[a^{\dagger}, a^{\dagger}\right]=[a, a]=0$. 
Among all density matrices $\rho$, the so-called quasi-free or Gaussian states are of particular interest 19-27: as mentioned before, they can be easily produced in experiments in quantum optics. These states are defined by the property that the expectations $\langle W(z)\rangle=\operatorname{tr}[W(z) \rho]$ of the Weyl operators $W(z)=e^{z a+z^{*} a^{\dagger}}$ are in Gaussian form, i.e. they are exponentials of a quadratic form in the complex variable $z$ and its conjugate $z^{*}$.

Let us collect annihilation and creation operators in the column vector a and its hermitian conjugate row vector $\mathbf{a}^{\dagger} \equiv\left(a^{\dagger} a\right)$. Then Gaussian states are fully characterized by the $2 \times 2$ matrix of bilinear correlations $(\mu, \nu=1,2) ! 23-27$

$$
\mathbf{G}_{\mu \nu} \equiv\left\langle\mathbf{a}_{\mu} \mathbf{a}_{\nu}^{\dagger}\right\rangle=\operatorname{Tr}\left[\mathbf{a}_{\mu} \mathbf{a}_{\nu}^{\dagger} \rho\right]=\left(\begin{array}{cc}
\left\langle a a^{\dagger}\right\rangle & \langle a a\rangle \\
\left\langle a^{\dagger} a^{\dagger}\right\rangle & \left\langle a^{\dagger} a\right\rangle
\end{array}\right) \equiv\left(\begin{array}{cc}
\beta & \alpha \\
\alpha^{*} & \beta-1
\end{array}\right) .
$$

From its definition, it follows that the matrix $\mathbf{G}$ in (11) is non-negative: $\mathbf{G} \geq 0$, so that $\beta \geq 1$ and $\beta(\beta-1) \geq|\alpha|^{2}$; one can show that this is also a sufficient condition for the state $\rho$ to represent a physical state ${ }^{19}$ For simplicity, we henceforth assume $\left\langle a^{\dagger}\right\rangle=\langle a\rangle=0$; this condition can be easily released, by taking $\langle W(z)\rangle$ to include exponentials of linear terms in $z$ and $z^{*}$.

As mentioned in the introductory remarks, our analysis is based on the assumption that the time evolution of a single oscillator immersed in a bath be Markovian and given by a semigroup, i.e. by a trace preserving, one parameter family of linear maps, acting on the set of density matrices $\rho$ representing the oscillator states. These maps are generated by master equations of the following Kossakowski-Lindblad form: $1-5$

$$
\frac{\partial \rho(t)}{\partial t}=-i[H, \rho(t)]+L[\rho(t)]
$$

with $H$ an effective Hamiltonian; instead, $L$ is a linear map representing the dissipative piece: it describes the effects of noise induced by the presence of the environment. The semigroup evolutions that preserve the set of Gaussian states are generated by equation in form (2) with $H$ and $L$ that are quadratic in the creation and annihilation operators, $27-29$

$$
\begin{aligned}
& H=\frac{1}{2} \omega\left\{a^{\dagger}, a\right\}, \quad \omega \geq 0 \\
& L[\rho]=\eta\left(\left[a \rho, a^{\dagger}\right]+\left[a, \rho a^{\dagger}\right]\right)+\sigma\left(\left[a^{\dagger} \rho, a\right]+\left[a^{\dagger}, \rho a\right]\right) \\
& -\lambda^{*}[a,[a, \rho]]-\lambda\left[a^{\dagger},\left[a^{\dagger}, \rho\right]\right] ;
\end{aligned}
$$

the coefficients $\eta, \sigma$ and $\lambda$ encode the physical properties of the environment and can be expressed in terms of the Fourier transform of the correlation functions in the bath! $1-5$

By using the two-dimensional vectors $\mathbf{a}$ and $\mathbf{a}^{\dagger}$ introduced before, the dissipative term in (3) can be recast in the following compact form:

$$
L[\rho]=\sum_{\mu, \nu=1}^{2} \mathbf{C}_{\mu \nu}\left(\mathbf{a}_{\nu} \rho \mathbf{a}_{\mu}^{\dagger}-\frac{1}{2}\left\{\mathbf{a}_{\mu}^{\dagger} \mathbf{a}_{\nu}, \rho\right\}\right),
$$


where the bath coefficients are now embedded in the $2 \times 2$ Kossakowski matrix:

$$
\mathbf{C}=\left(\begin{array}{cc}
\eta & \lambda^{*} \\
\lambda & \sigma
\end{array}\right)
$$

The dissipative parameters in (5) are not completely arbitrary. First of all, $\eta$ and $\sigma$ need to be real in order to comply with the hermiticity preserving requirement of the generated semigroup. In addition, the request that the finite-time evolution $\gamma_{t}$ generated by a master equation of the form (2), (3) map physical states into physical states, i.e. preserve the positivity of the density matrix $\rho(t)$ for all times, gives rise to further constraints.

The simplest way to guarantee the positivity of the dynamics $\gamma_{t}$ is to require the Kossakowski matrix in (5) to be non-negative

$$
\mathbf{C} \geq 0 \text {. }
$$

Indeed, this condition is equivalent to the requirement of complete positivity, a stronger property than simple positivity, that guarantees the physical consistency of the evolution $\gamma_{t}$ in all possible situations $1-\frac{1}{5}$ Dynamics of such type, obtained from the equation (2), with Hamiltonian as in (3) and dissipative term as in (4), (6), are known in the literature as quasi-free quantum dynamical semigroups: $27-29$ as already observed, they are characterized by the property of transforming the set of Gaussian states into itself.

Nevertheless, in phenomenological applications, the condition of complete positivity is often dismissed as unnecessary 34 , since in many naive derivations of the equation (2) generating the reduced dynamics $\gamma_{t}$, the hierarchy among the dissipative parameters imposed by the condition (6) appear not to be satisfied $13-16$ In such cases, it is easy to show that the resulting dynamics $\gamma_{t}$ is not positive, hence physically unacceptable.

In order to explicitly show this, it is convenient to consider the time evolution of the correlations in (11), as induced by the dynamics of the density matrix $\rho(t)$, through the duality map: $\operatorname{Tr}\left[\mathbf{a}_{\mu} \mathbf{a}_{\nu}^{\dagger} \rho(t)\right]=\operatorname{Tr}\left[\left(\mathbf{a}_{\mu} \mathbf{a}_{\nu}^{\dagger}\right)(t) \rho\right]$. One explicitly finds: $27-29$

$$
\partial_{t} \mathbf{G}(t)=\mathbf{A}^{\dagger} \cdot \mathbf{G}(t)+\mathbf{G}(t) \cdot \mathbf{A}+\mathbf{B},
$$

where the $2 \times 2$ matrices $\mathbf{A}$ and $\mathbf{B}$ contain the dependence on the hamiltonian $\omega$ and dissipative parameters $\eta, \sigma$ and $\lambda$ :

$$
\mathbf{A}=\frac{1}{2}\left(\begin{array}{cc}
\sigma-\eta+2 i \omega & 0 \\
0 & \sigma-\eta-2 i \omega
\end{array}\right) \quad \mathbf{B}=\left(\begin{array}{cc}
\eta & -\lambda^{*} \\
-\lambda & \sigma
\end{array}\right) .
$$

The positivity condition $\mathbf{G}(t) \geq 0$ needs to be preserved for all times in order for the the evolution $\gamma_{t}: \rho(0) \rightarrow \rho(t)$ to be positive and thus physically acceptable. In order to prove that this is not the case when (6) is not satisfied, i.e. when $\eta \sigma<|\lambda|^{2}$, it is sufficient to prove that $\mathbf{G}\left(t^{\prime}\right)<0$, for a certain $t^{\prime}$. Let us assume that indeed this is the case; since we start with a physical state, i.e. $\mathbf{G}(0) \geq 0$, there will be 
a time $t^{\prime \prime}<t^{\prime}$ for which $\mathbf{G}\left(t^{\prime \prime}\right)$ has a null eigenvalue. Since we are dealing with evolutions of semigroup form, without loss of generality, we can set $t^{\prime \prime}=0$.

Let us now consider the average $\mathbf{Q}(t) \equiv\langle\Psi|\mathbf{G}(t)| \Psi\rangle$, where the two-component vector $|\Psi\rangle$ is the eigenvector of $\mathbf{G}(0)$ corresponding to its null eigenvalue. Recalling (11), one easily finds $\langle\Psi|=\left(-\alpha^{*}, \beta\right)$, with the condition $\beta(\beta-1)=|\alpha|^{2}$, that assures the vanishing of the determinant of $\mathbf{G}(0)$. Clearly, since $\mathbf{Q}(0)=0, \mathbf{G}(t)$ will start acquiring a negative eigenvalue as soon as:

$$
\partial_{t} \mathbf{Q}(0)<0
$$

Using (7) and (8), this condition reduces to:

$$
\eta(\beta-1)+\sigma \beta+2 \mathcal{R} e(\alpha \lambda)<0 .
$$

Since $\eta, \sigma \geq 0$ and $\beta \geq 1$, in order to satisfy (10) one needs to choose the phase of $\alpha$ in such a way $\operatorname{Re}(\alpha \lambda)$ be large and negative, i.e. $\operatorname{Re}(\alpha \lambda)=-|\alpha||\lambda|$. Then, one can check that for $\eta>\sigma$, the condition (10) is always satisfied; this remains true also in the case $\eta \leq \sigma$, provided $|\lambda|<\sigma$. As a consequence, when the Kossakowski matrix in (5) is negative, the evolution generated by (2), (3) results non-positive, and therefore physically unacceptable.

As mentioned in the Introduction, in order to cure this pathology and continue to use semigroups of non-positive maps to model reduced dynamics, an ad hoc prescription has been proposed $14-16$ restrict the possible initial states $\rho(0)$ to those for which $\rho(t)=\gamma_{t}[\rho(0)], t>0$, as generated by (2), (3), remains a physical Gaussian state. The argument is that any Markov approximation neglects a certain initial span of time, namely a transient, during which no semigroup time-evolution is possible due to unavoidable memory effects. The transient dynamics can be effectively depicted as a slippage operator that, out of all possible initial states, selects those which do not conflict with the Markov time-evolution when it sets in. As we shall see in the next section, the slippage-argument may cure the positivity-preserving problem, but appears to be inconclusive when dealing with bi- and multi-partite open quantum systems because of the existence of entangled states.

\section{Two-Mode Dynamics and Entanglement}

We shall now extend the treatment discussed so far to the case of two oscillators, one of which still immersed in an external environment and therefore evolving with the dissipative, semigroup dynamics generated by (2), (3), while the other undergoes a unitary evolution. As mentioned in the Introduction, such a system can actually describe physically implementable situations in quantum optics, e.g. that of polarized, coherent states propagating along two fibers, one which is subjected to noise, while the other is not. Having a physically consistent theoretical treatment of the behavior of such setups is therefore of great phenomenological relevance.

In this case of bipartite, two-mode settings, Gaussian states $\rho$ are still characterized by having expectation $\left\langle W\left(z_{1}, z_{2}\right)\right\rangle=\operatorname{tr}\left[W\left(z_{1}, z_{2}\right) \rho\right]$ of the Weyl operators 
$W\left(z_{1}, z_{2}\right)=e^{z_{1} a_{1}+z_{2} a_{2}+z_{1}^{*} a_{1}^{\dagger}+z_{2}^{*} a_{2}^{\dagger}}$ in Gaussian form. These states are again fully characterized by the correlation matrix $\mathbf{G}$, which is now $4 \times 4$, and still defined as in (1). The column vector $\mathbf{a}$ and its conjugate $\mathbf{a}^{\dagger}$ are now four-dimensional, with components $\mathbf{a}_{\mu}^{\dagger}=\left(a_{1}^{\dagger}, a_{1}, a_{2}^{\dagger}, a_{2}\right), \mu=1,2,3,4$, where $a_{i}^{\dagger}, a_{i}, i=1,2$, are single mode creation and annihilation operators, obeying the standard oscillator algebra: $\left[a_{i}, a_{j}^{\dagger}\right]=\delta_{i j},\left[a_{i}^{\dagger}, a_{j}^{\dagger}\right]=0=\left[a_{i}, a_{j}\right]$.

The time evolution is again a Markovian semigroup generated by a master equation of the form (2). The Hamiltonian is now the sum of two pieces:

$$
H=\frac{1}{2} \omega_{1}\left\{a_{1}^{\dagger}, a_{1}\right\}+\frac{1}{2} \omega_{2}\left\{a_{2}^{\dagger}, a_{2}\right\},
$$

while the dissipative contribution can be written as in (4), with a $4 \times 4$ Kossakowski matrix with null entries, except in the upper left corner where it coincides with (5). Such compound dynamics $\Gamma_{t}=\gamma_{t} \otimes U_{t}$, being represented by the tensor product of the dissipative evolution $\gamma_{t}$ discussed in the previous section for the first oscillator mode and of a unitary one $U_{t}$ for the second mode, preserves the Gaussian form of the states $\rho$.

As in the single mode case, the time evolution equation can be equivalently formulated in terms of the correlation matrix; explicitly:

$$
\partial_{t} \mathbf{G}(t)=\mathcal{A}^{\dagger} \cdot \mathbf{G}(t)+\mathbf{G}(t) \cdot \mathcal{A}+\mathcal{B},
$$

where $\mathcal{A}$ is diagonal with entries $\frac{1}{2}\left(\sigma-\eta+2 i \omega_{1}, \sigma-\eta-2 i \omega_{1}, 2 i \omega_{2},-2 i \omega_{2}\right)$, while the inhomogeneous term is given by

$$
\mathcal{B}=\left(\begin{array}{ll}
\mathbf{B} & \mathbf{0} \\
\mathbf{0} & \mathbf{0}
\end{array}\right)
$$

with $\mathbf{0}$ representing the $2 \times 2$ zero matrix and $\mathbf{B}$ is as in (8). The matrix $\mathbf{G}(\mathbf{t})$ can similarly be written in block form:

$$
\mathbf{G}(t)=\left(\begin{array}{cc}
\mathbf{G}_{1}(t) & \mathbf{W}(t) \\
\mathbf{W}^{\dagger}(t) & \mathbf{G}_{2}(t)
\end{array}\right)
$$

where $\mathbf{G}_{1}(t)$ and $\mathbf{G}_{2}(t)$ are $2 \times 2$ correlation matrices corresponding to the reduced single-mode Gaussian states obtained by tracing over the degrees of freedom of the second, $\operatorname{Tr}_{2}[\rho(t)]$, respectively first, $\operatorname{Tr}_{1}[\rho(t)]$, oscillator. As such, they must satisfy the positivity condition: $\mathbf{G}_{i}(t) \geq 0, i=1,2$, for all times.

Therefore, to avoid appearance of negative probabilities when the single mode dynamics $\gamma_{t}$ is not positive, i.e. when $\eta \sigma<|\lambda|^{2}$, in line with the "slippage" prescription, one has to restrict the choice of the initial condition $\rho(0)$ to those Gaussian density matrices for which the reduced states $\operatorname{Tr}_{2}[\rho(0)]$ gives rise to positive correlation matrices, $\mathbf{G}_{1}(t) \geq 0$. Although this prescription clearly works for initial states in factorized form, it fails in general when $\rho(0)$ is entangled.

In order to make the incompatibility between slippage and entanglement apparent, let us consider an initial correlation matrix of the form:

$$
\mathbf{G}(0)=\left(\begin{array}{cc}
\mathbf{G}_{1} & \mathbf{G}_{1}^{1 / 2} \mathbf{G}_{2}^{1 / 2} \\
\mathbf{G}_{2}^{1 / 2} \mathbf{G}_{1}^{1 / 2} & \mathbf{G}_{2}
\end{array}\right)
$$


with the conditions $\mathbf{G}_{i} \geq 0, i=1,2$; note that the matrix in (15) satisfy the physical condition $\mathbf{G}(0) \geq 0$ by construction. Further, the $2 \times 2$ correlation submatrices $\mathbf{G}_{i}$ are chosen in such a way that their positivity is preserved under the action of the compound dynamics $\Gamma_{t}$. Let $\mathbf{G}_{2}$ be invertible. Then, the $4 \times 4$ matrix in (15) possesses a zero eigenvalue, whose associated eigenvector can be written as

$$
|\Psi\rangle=\left(\begin{array}{c}
|\psi\rangle \\
-\mathbf{G}_{2}^{-1 / 2} \mathbf{G}_{1}^{1 / 2}|\psi\rangle
\end{array}\right),
$$

where $|\psi\rangle$ is an arbitrary two-dimensional vector. Let us then consider the average $\mathcal{Q}(t) \equiv\langle\Psi|\mathbf{G}(t)| \Psi\rangle$. Since $\mathcal{Q}(0)=0$, the matrix $\mathbf{G}(t)$ will start developing negative eigenvalues as soon as

$$
\partial_{t} \mathcal{Q}(0)<0
$$

Explicitly, $\partial_{t} \mathcal{Q}(0)=\langle\Psi|\mathcal{B}| \Psi\rangle=\langle\psi|\mathbf{B}| \psi\rangle$; therefore, the condition (17) can always be satisfied by choosing for the two-vector $|\psi\rangle$ the negative eigenvector of $\mathbf{B}$.

The physical inconsistency of dynamics for which the Kossakowski matrix in (5) is not positive is now apparent: the slippage prescription make them positive, but not completely positive; therefore, when the subsystem is bipartite, one can always find an entangled Gaussian state that is mapped out from the set of physical states by the compound dynamics $\Gamma_{t}$.

That the initial state $\rho(0)$ giving rise to the correlations in (15) is entangled can be directly verified using the operation of partial transposition $\frac{35 \mid 36}{2}$, i.e. of transposition involving only one of the two oscillators, say the second one. It can be most easily implemented on symmetric correlation matrices:

$$
\mathbf{V}_{\mu \nu}=\frac{1}{2}\left\langle\left\{\mathbf{a}_{\mu}, \mathbf{a}_{\nu}^{\dagger}\right\}\right\rangle \equiv \frac{1}{2} \operatorname{Tr}\left[\left(\mathbf{a}_{\mu} \mathbf{a}_{\nu}^{\dagger}+\mathbf{a}_{\nu}^{\dagger} \mathbf{a}_{\mu}\right) \rho\right]
$$

where it amounts to the exchange $a_{2} \leftrightarrow a_{2}^{\dagger}$. This transformation clearly maps states into states for separable ones, but not in general for correlated ones: it thus provides a sufficient criterion for bipartite entanglement, which turns out to be also sufficient for two-mode states, as in in the present case. 37

For the case at hand, one finds the the operation of partial transposition with respect to the second system results in the following transformation of the $4 \times 4$ symmetric covariance:

$$
\mathbf{V} \rightarrow \widetilde{\mathbf{V}}=\mathbf{T} \cdot \mathbf{V} \cdot \mathbf{T}, \quad \mathbf{T}=\left(\begin{array}{cc}
\mathbf{1} & \mathbf{0} \\
\mathbf{0} & \sigma_{1}
\end{array}\right)
$$

where 1 is the $2 \times 2$ unit matrix, while $\sigma_{1}$ is the first Pauli matrix. Equivalently, for $\mathbf{G}(0)$ in (15), one finds:

$$
\mathbf{G}(0) \rightarrow \widetilde{\mathbf{G}}(0)=\left(\begin{array}{cc}
\mathbf{G}_{1} & \mathbf{G}_{1}^{1 / 2} \mathbf{G}_{2}^{1 / 2} \sigma_{1} \\
\sigma_{1} \mathbf{G}_{2}^{1 / 2} \mathbf{G}_{1}^{1 / 2} & \widetilde{\mathbf{G}}_{2}
\end{array}\right)
$$


where $\widetilde{\mathbf{G}}_{2}$ indicates transposition of $\mathbf{G}_{2}$. By construction, the initial density matrix $\rho(0)$ corresponds to a physical state, i.e. $\mathbf{G}(0) \geq 0$; if one further finds:

$$
\widetilde{\mathbf{G}}(0)<0,
$$

then the state $\rho(0)$ is surely entangled. One can show that (21) is equivalent to the following condition involving $2 \times 2$ submatrices of $\mathbf{G}(0) ! 38$

$$
\mathbf{G}_{1}-\mathbf{G}_{1}^{1 / 2} \mathbf{G}_{2}^{1 / 2}\left[\widetilde{\mathbf{G}}_{2}\right]^{-1} \mathbf{G}_{2}^{1 / 2} \mathbf{G}_{1}^{1 / 2}<0,
$$

which in turn reduces to:

$$
\left[\mathbf{G}_{2}\right]^{-1}-\sigma_{1}\left[\widetilde{\mathbf{G}}_{2}\right]^{-1} \sigma_{1}<0
$$

Since $\mathbf{G}_{2}$ can always be written as in (1), with $\beta(\beta-1)>|\alpha|^{2}$, by direct computation, one immediately finds that the combination in the l.h.s. of (23) always possesses a negative eigenvalue, and therefore that $\rho(0)$ is indeed an entangled state.

\section{Discussion}

The dynamics of a subsystem in weak interaction with an external environment can be described in terms of semigroups of linear maps $\gamma_{t}$ generated by a Markovian master equation. Although rigorously proven only in special cases, this result is nevertheless believed to hold in general, since memory effects should disappear from the reduced subsytem dynamics as soon as all correlations in the environment have died out.

This mathematical effective description of reduced dissipative dynamics needs to satisfy further physical constraints. In the first place, it must preserve the positivity of any initial density matrix: only in this case, the time-evolution turns out to be consistent with the interpretation of the state-eigenvalues as probabilities. In the second place, one has also to care of possible couplings with another system, not subjected to noise, and therefore to guarantee the positivity-preserving character also of the semigroup of maps of the form $\Gamma_{t}=\gamma_{t} \otimes U_{t}$, as studied in the previous section; this is only assured in all possible situations if $\gamma_{t}$ is completely positive.

Nevertheless, in many applications, complete positivity is often dismissed as unnecessary. This attitude is based on the fact that this property guarantees full physical consistency of the reduced dynamics at the price of order relations among the parameters describing dissipation ( $c f$. (6) $)$, which may appear to have little physical motivations. Further, most phenomenological derivation of reduced dissipative dynamics lead to semigroup of linear transformation that are not completely positive.

Unfortunately, such derivations often lead to time evolutions that are not even positive. To avoid inconsistencies, one usually restricts the possible initial states to those for which $\gamma_{t}$ remains positive (the so-called "slippage of initial conditions"). This prescription works also in the case of the evolution $\Gamma_{t}$ for two subsystems, provided the initial state is in separable form: $\rho(0)=\sum_{i} p_{i} \rho_{i}^{(1)} \otimes \rho_{i}^{(2)}, p_{i} \geq 0$, 
$\sum_{i} p_{i}=1$, where $\rho_{i}^{(1)}$ and $\rho_{i}^{(2)}$ are admissible states for the first and second subsystems, respectively.

On the contrary, as shown in the previous section, when the initial state $\rho(0)$ is not in factorized form, the evolved matrix $\rho(t)=\Gamma_{t}[\rho(0)]$ fails to be positive for all times. In keeping with the same attitude adopted for a single subsystem dynamics $\gamma_{t}$, to cure this additional inconsistency one has to further restrict the domain of applicability of $\Gamma_{t}$. However, this is again a temporary solution; indeed, the whole discussion needs be repeated when three or more subsystems are considered.

These considerations seems to suggest that there is an intrinsic incompatibility between the existence of entangled states and the slippage prescription adopted to cure the inconsistencies that non-completely positive, or even non-positive, reduced dynamics might produce. Note that this conclusion can not be dismissed as being purely academic; on the contrary, it seems to have a direct experimental relevance: as mentioned before, the scenario studied in the previous sections might have an actual realization in quantum optics. From this perspective, the widely used cure of redefining the initial conditions in case of non-positive Markovian dynamics does not appear to be completely satisfactory.

\section{References}

1. R. Alicki, K. Lendi, Quantum Dynamical Semi-Groups and Applications, Lect. Notes Phys. 286, (Springer-Verlag, Berlin, 1987)

2. E.B. Davies, Quantum Theory of Open Systems, (Academic Press, New York, 1976)

3. V. Gorini, A. Frigerio, M. Verri, A. Kossakowski and E.C.G. Surdarshan, Rep. Math. Phys. 13 (1978) 149

4. H. Spohn, Rev. Mod. Phys. 53 (1980) 569

5. F. Benatti and R. Floreanini, Int. J. Mod. Phys. B 19, 3063 (2005)

6. Dissipative Quantum Dynamics, F. Benatti and R. Floreanini, Eds., Lect. Notes Phys. 622, (Springer-Verlag, Berlin, 2003)

7. W.H. Louisell, Quantum Statistical Properties of Radiation, (Wiley, New York, 1973)

8. C.P. Slichter, Principle of Magnetic Resonance (Springer-Verlag, Berlin, 1990)

9. C.W. Gardiner and P. Zoller, Quantum Noise, II ed., (Springer, Berlin, 2000)

10. M.O. Scully and M.S. Zubairy, Quantum Optics (Cambridge University Press, Cambridge, 1997)

11. R.R. Puri, Mathematical Methods of Quantum Optics, (Springer, Berlin, 2001)

12. H.-P. Breuer and F. Petruccione, The Theory of Open Quantum Systems (Oxford University Press, Oxford, 2002)

13. S. Gnutzmann and F. Haake, Z. Phys. B 10 (1996) 263

14. A. Suarez, R. Silbey and I. Oppenheim, J. Chem. Phys. 97 (1992) 5101

15. P. Gaspard and M. Nagaoka, J. Chem. Phys. 111 (1999) 5668

16. J. Wielkie, J. Chem. Phys. 114 (2001) 7736

17. F. Benatti, R. Floreanini and M. Piani, Phys. Rev. A 67 (2003) 042110

18. F. Benatti, R. Floreanini and S. Breteaux, Slipped non-positive reduced dynamics and entanglement, preprint, 2006

19. A.S. Holevo, Probabilistic and Statistical Aspects of Quantum Theory, (North Holland, Amsterdam, 1982)

20. G. Lindblad, J. Phys. A 33 (2000) 5059 
21. J. Eisert and M.B. Plenio, Int. J. Quant. Inf. 1 (2003) 479

22. B.-G. Englert and K. Wódkiewicz, Int. J. Quant. Inf. 1 (2003) 153

23. A. Serafini, M. Paris, F. Illuminati and S. De Siena, J. Opt. B 7 (2005) R19

24. A. Ferraro, S. Olivares, M. Paris, Gaussian States in Continuous Variable Quantum Information, (Bibliopolis, Napoli, 2005)

25. S.L. Braunstein and P. van Loock, Rev. Mod. Phys. 77 (2005) 513

26. F. Benatti and R. Floreanini, J. Phys. A 33 (2000) 8139

27. F. Benatti and R. Floreanini, J. Phys. A 39 (2006) 2689

28. B. Demoen, P. Vanheuverzwijn and A. Verbeure, Rep. Math. Phys. 15 (1979) 27

29. P. Vanheuverzwijn, Ann. Inst. H. Poncaré A29 (1978) 123

30. E. Collett, Polarized Light, (Marcel Dekker, New York, 1993)

31. S. Huard, Polarization of Light, (Wiley, New York, 1997)

32. C. Brosseau, Fundamentals of Polarized Light, (Wiley, New York, 1998)

33. F. Benatti and R. Floreanini, Tests of complete positivity in fiber optics, preprint, 2006

34. P. Pechukas, Phys. Rev. Lett. 73, 1060 (1994)

35. A. Peres, Phys. Rev. Lett. 77 (1996) 1413

36. M. Horodecki, P. Horodecki and R. Horodecki, Phys. Lett. A 223 (1996) 1

37. R. Simon, Phys. Rev. Lett. 84 (2000) 2726

38. G. Giedke, B. Kraus, M. Lewenstein and J.I. Cirac, Phys. Rev. Lett. 87 (2001) 167904 